\title{
Article \\ Receding Contact Problem of Multi-Layered Elastic Structures Involving Functionally Graded Materials
}

\author{
Jie Yan ${ }^{1,2}$ and Cong Wang ${ }^{1, *}$
}

1 Intelligent Policing Key Laboratory of Sichuan Province, Sichuan Police College, Luzhou 646000, China; yan_jie@foxmail.com

2 Jiangsu Key Laboratory of Engineering Mechanics, School of Civil Engineering, Southeast University, Nanjing 210096, China

* Correspondence: cong-wang@foxmail.com

check for updates

Citation: Yan, J.; Wang, C. Receding Contact Problem of Multi-Layered Elastic Structures Involving Functionally Graded Materials. Crystals 2022, 12, 354. https:// doi.org/10.3390/cryst12030354

Academic Editors: Gongye Zhang, Shuitao Gu, Shuohui Yin and Abdolhamid Akbarzadeh Shafaroudi

Received: 24 January 2022 Accepted: 25 February 2022 Published: 6 March 2022

Publisher's Note: MDPI stays neutral with regard to jurisdictional claims in published maps and institutional affiliations.

Copyright: (c) 2022 by the authors. Licensee MDPI, Basel, Switzerland. This article is an open access article distributed under the terms and conditions of the Creative Commons Attribution (CC BY) license (https:// creativecommons.org/licenses/by/ $4.0 /)$.

\begin{abstract}
This paper studies a receding contact problem of a functionally graded layer laminate pressed against a functionally graded coated homogeneous half-plane substrate by a rigid flat indenter. The shear modulus of the functionally graded materials with a constant Poisson's ratio is modeled by an exponential function which varies along the thickness direction. Both the governing equations and the boundary conditions of the receding contact problem are converted into a pair of singular integral equations using the Fourier integral transforms, which are numerically integrated by the Chebyshev-Gauss quadrature. The contact pressure and the contact size at both contact interfaces are eventually obtained iteratively, as developed from the steepest descent algorithm. Extensive parametric studies suggest that it is possible to regulate the contact pressure and contact size by constructing the top layer from a soft functionally graded material.
\end{abstract}

Keywords: receding contact; functionally graded material (FGM); singular integral equation

\section{Introduction}

For a multi-layered structure, according to the variation of the contact size between different layers under an external load, three contact types can be identified: advancing contact, if the contact size becomes larger; Stationary contact, if the contact size remains unchanged; and receding contact, if the contact size becomes smaller. Among these, receding contact has not been well studied, since it rarely occurs in a single-layer structure. For receding contact, however, a smaller contact size means more concentrated pressure. Hence, for multi-layered structures, receding contact may give rise to several adverse effects, including layer peeling, delamination fracture, etc.

A variety of methods have been adopted to study the receding contact problems of multi-layered homogeneous elastic structures. By using Fourier transform method, Comez et al. [1] studied the receding contact problem of double elastic layers indented by a rigid cylindrical indenter. On the basis of this work, Cakiroglu et al. [2] introduced the artificial neural networks method to reproduce the problem. By using Fourier transform method, Adibelli et al. [3] studied the receding contact problem between a coated layer and a half-plane loaded by a rigid cylindrical indenter. Yaylaci and Birinci [4] analyzed a double receding contact problem between two elastic layers and two elastic quarter-planes by using an integral transform technique, further verified by the finite element method [5] and the artificial neural network method [6].

Functionally graded materials (FGM) are generally formed from several inhomogeneous particulate composites in such a way that the volume fractions of the constituents vary along any desired spatial direction. Special preparations can yield FGM gradient changes along the thickness or in any other directions. In theoretical studies, linear functions $[7,8]$, exponential functions $[9,10]$, and power functions $[11,12]$ are all used to fit the variations of the material properties approximately. Among these, the exponential function 
family is the principal choice for a good fitting capacity, since different variation features can be approximated by changing the exponential parameter.

In the past twenty years, advances in the field of materials science have enabled that the FGMs serve as a better alternative to conventional homogeneous coatings or layers [13]. For fulfilling specific industrial applications, FGMs have been widely used in structural elements such as beams, plates, and shells [14,15]. The preparation technology of FGMs is continually being improved [16-18], and the research on the microstructure [19-21], fracture [22-24], and mechanical properties [24-27] of functionally graded materials is gradually catching up.

Currently, the receding contact problems of FGM on a nano-scale also raise concerns. Choi and Paulino [28] study the thermoelastic contact between a rigid flat indenter and an FGM layer, and the thermal conductivity coefficients, shear moduli, and thermal expansion coefficients are all be fitted exponentially. Ke et al. [29] investigate the smooth contact problems of the piezoelectric FGM layer under several kinds of indenters. The partial slip contact between the FGM layers is also considered by Chen et al. [30].

The receding contact problems of multi-layered elastic structures involving FGM also attracted extensive attention. The frictionless/frictional receding contact of bi-FGlayers were studied successively [31,32]. Then, the receding contact difficulties between an FGM-coated layer and a homogeneous half-plane pressed by a rigid cylindrical indenter were analyzed [33,34]. The same receding contact structures loading by a distributive compressive traction field obtained both semi-analytical and finite element solutions $[35,36]$. Çömez et al. [37] investigated the problem of frictional receding contact of an FG layer resting on a homogeneously coated half-plane. Recently, Yaylac1 et al. [38] used the semianalytical method, the finite element method (FEM), and the multi-layer perceptron method to analyze the receding contact problem between an FG layer and a homogeneous elastic layer resting on a Winkler foundation which loaded with a rigid cylindrical indenter. Öner et al. [39] used the semi-analytical method, FEM, and an artificial neural network to study the double receding contact problem of multi-layered FGM structures under a distributive compressive traction field.

The above studies only focus on a rigid circular indenter or a uniform load. For the complexities introduced by the singularities of flat indenters, current studies of the receding contact problems involving a flat indenter continue to consider only bi-layer structures. Karabulut et al. [40] analyzed the receding contact problem of a layer resting on a half-plane due to two flat-ended indenters. Ozsahin and TaskJner [41] investigated an elastic layer on an elastic half plane loaded by three rigid flat indenters. A recent study [42] solved for the frictional receding contact between an FG layer and an orthotropic substrate under the indentation of a rigid flat-ended indenter, and Cao and $\mathrm{Mi}$ [43] investigated the receding contact between a graded and a homogeneous layer.

Adding to the challenge, the triple-layer structure requires a higher computation accuracy and speed and additionally, singularity at the endpoint of the flat indenter sharply raises the difficulty level. To our knowledge, there is no study that considers the receding contact problem of a triple-layer structure loaded with a flat indenter. In this paper, a receding contact problem for an FG layer resting on an FGM coated half-plane due to a flat indenter is studied semi-analytically. The remainder of this paper is organized as follows: In Section 2, the mathematical formulation of the receding contact problem is outlined in detail. In Section 3, considering the tip singularity of the flat indenter simultaneously, the first and second types of Gauss-Chebyshev integral formulas are used in dealing with the singular integral equations, coupled with different types of Chebyshev polynomials in order to discretize the equations, and then an iterative numerical solution is derived. In Section 4, a variety of numerical experiments detailing the pressure distributions on both contact surfaces are reported. Finally, several useful conclusions are drawn in Section 5. 


\section{Materials and Methods}

In a multi-layered structure like that shown in Figure 1, the contact surface lying between the FG layers may be partially separate, while a rigid flat indenter with a halflength presses into the top layer. Figure 1 illustrates a typical scenario in some industry applications. Taking the punching machine as an example, the upper FG layer represents an FG gasket, and the lower FG layer represents the FG-coatings established for abating the shock effect on the substrate. The thicknesses of the FG layers are respectively $h_{2}$ and $h_{3}$. The shear modulus of the half-plane substrate is treated as a constant $\mu_{1}$, and both the FG layers are modeled by exponential functions varying along their thickness dimensions:

$$
\begin{gathered}
\mu_{3}=\mu_{03} e^{\beta_{3} y},\left(h_{2}<y<h_{3}+h_{2}\right), \\
\mu_{2}=\mu_{1} e^{\beta_{2} y},\left(0<y<h_{2}\right),
\end{gathered}
$$

where $\beta_{2}$ and $\beta_{3}$ are arbitrary non-zero constants, and $\mu_{03}$ represents the shear modulus of the top FG layer's bottom surface. The Poisson's ratios of the FG layers are both fixed as the same constant $v$, since the value can hardly influence the contact substantially [44]. To simplify the computation, this work also ignores the influences of the gravitational and frictional forces. Under the proposed assumptions, each layer's boundary condition can be enumerated as:

$$
\begin{gathered}
\sigma_{y y 3}\left(x, h_{2}+h_{3}\right)=-p(x) H(a-|x|),(|x|<+\infty) ; \\
\sigma_{x y 3}\left(x, h_{2}+h_{3}\right)=0,(|x|<+\infty) ; \\
\sigma_{y y 3}\left(x, h_{2}\right)=-q(x) H(b-|x|),(|x|<+\infty) ; \\
\sigma_{x y 3}\left(x, h_{2}\right)=0,(|x|<+\infty) ; \\
\sigma_{y y 2}\left(x, h_{2}\right)=-q(x) H(b-|x|),(|x|<+\infty) ; \\
\sigma_{x y 2}\left(x, h_{2}\right)=0,(|x|<+\infty) ; \\
\sigma_{y y 1}(x, 0)=\sigma_{y y 2}(x, 0),(|x|<+\infty) ; \\
\sigma_{x y 1}(x, 0)=\sigma_{x y 2}(x, 0)=0,(|x|<+\infty) ; \\
u_{1}(x, 0)=u_{2}(x, 0),(|x|<+\infty) ; \\
v_{1}(x, 0)=v_{2}(x, 0),(|x|<+\infty) .
\end{gathered}
$$

where $H$ denotes the Heaviside step function, and $\sigma_{x x j}, \sigma_{x y j}$ and $\sigma_{y y j}$ represent the pressure components of the $j$-th layer $(j=1,2,3)$ respectively, $p(x)$ represents the distribution function of the stationary contact pressure, and $q(x)$ represents the distribution function of the receding contact pressure, $u_{j}$ and $v_{j}$ represent the displacement components of the $j$-th layer $(j=1,2,3)$.

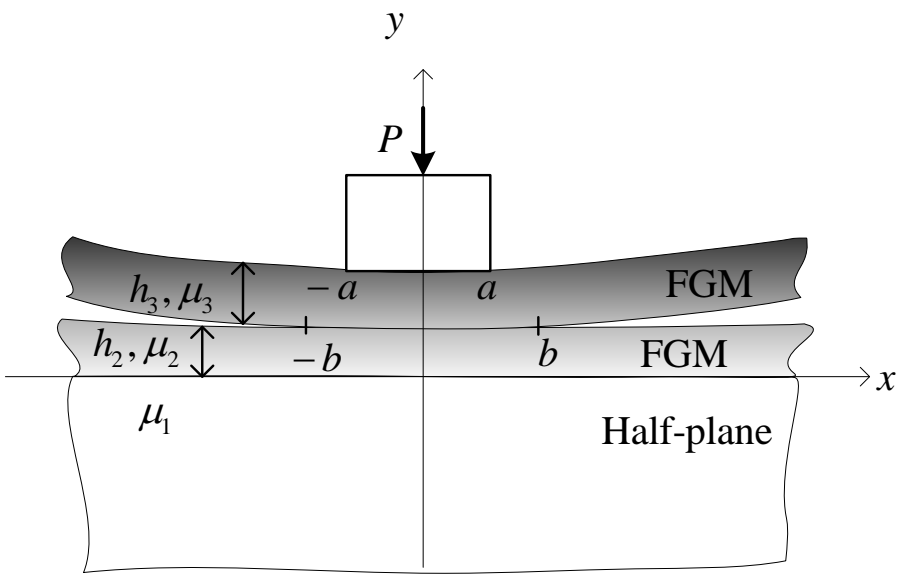

Figure 1. Geometry of the presented receding contact problem. 
For the absence of the frictional forces, the vertical displacement certainly stays continuous in both contact areas. To ensure the continuity of the vertical displacement and exclude the unwanted effects of rigid-body displacement, this work quantifies the profile of the rigid indenter by its slope function $f(x)=0$. Taken together, these conditions indicate that:

$$
\begin{gathered}
\frac{\partial}{\partial x}\left[v_{3}\left(x, h_{2}+h_{3}\right)\right]=0,(|x| \leq a), \\
\frac{\partial}{\partial x}\left[v_{1}(x, 0)-v_{2}(x, 0)\right]=0,(|x| \leq b) .
\end{gathered}
$$

According to the basic formulas of mechanics (the equilibrium equation, the straindisplacement relationships, and the linear elastic stress-strain law), the following equations concerning the displacements of layers are satisfied:

$$
\begin{gathered}
\frac{\kappa+1}{\kappa-1} \frac{\partial^{2} u_{j}}{\partial x^{2}}+\frac{\partial^{2} u_{j}}{\partial y^{2}}+\frac{2}{\kappa-1} \frac{\partial^{2} v_{j}}{\partial x \partial y}+\beta_{j} \frac{\partial u_{j}}{\partial y}+\beta_{j} \frac{\partial v_{j}}{\partial x}=0 \\
\frac{\kappa-1}{\kappa+1} \frac{\partial^{2} v_{j}}{\partial x^{2}}+\frac{\partial^{2} v_{j}}{\partial y^{2}}+\frac{2}{\kappa+1} \frac{\partial^{2} u_{j}}{\partial x \partial y}+\frac{(3-\kappa) \beta_{j}}{\kappa+1} \frac{\partial u_{j}}{\partial x}+\beta_{j} \frac{\partial v_{j}}{\partial y}=0,(\mathrm{j}=1,2,3),
\end{gathered}
$$

where $\beta_{1}=0$, the above equations are suitable for homogeneous half-plane; Kolosov's constant $\kappa$ is related to the Poisson's ratio by $\kappa=3-4 v$ for plane strain and $\kappa=(3-v) /(1+v)$ for plane stress.

To address the formal solutions for the displacements and pressure components, this study maps the boundary conditions (3-12) into Fourier space using Fourier integral transform. The details can be obtained in our previous work [45]. By substituting the displacement components' integral expressions into Equations (13) and (14), the following system of singular integral equations can be obtained by the inverse Fourier transformation:

$$
\begin{aligned}
& \int_{-a}^{a}\left(\frac{1}{t-x}+K_{1}(x, t)\right) p(t) d t+e^{\beta_{2} h_{2}} \int_{-b}^{b} K_{2}(x, t) q(t) d t=0,(|x| \leq a), \\
& \int_{-a}^{a} K_{3}(x, t) p(t) d t+e^{\beta_{2} h_{2}} \int_{-b}^{b}\left(\frac{1}{t-x}+K_{4}(x, t)\right) q(t) d t=0,(|x| \leq b) .
\end{aligned}
$$

The expressions of $K_{j}(x, t)(j=1,2,3,4)$ are documented in Appendix A. The unknowns $b$ and the distribution function of the contact pressure should satisfy the equilibrium conditions of the force expressed by the following equations:

$$
\begin{aligned}
& \int_{-a}^{a} p(t) d t=\mathrm{P}, \\
& \int_{-b}^{b} q(t) d t=\mathrm{P},
\end{aligned}
$$

where $\mathrm{P}$ is the known resultant force applied to the flat indenter.

\section{Solution to Dual Singular Integral Equations}

To simplify the computation, all the physics qualities are non-dimensionalized by introducing the following transformations:

$$
r=\frac{x}{a},
$$




$$
\begin{aligned}
& r^{\prime}=\frac{x}{b^{\prime}} \\
& s=\frac{t}{a}, \\
& s^{\prime}=\frac{t}{b^{\prime}} \\
& \bar{K}_{1}(r, s)=K_{1}(x, t), \\
& \bar{K}_{2}\left(r, s^{\prime}\right)=K_{2}(x, t), \\
& \bar{K}_{3}\left(r^{\prime}, s\right)=K_{3}(x, t), \\
& \bar{K}_{4}\left(r^{\prime}, s^{\prime}\right)=K_{4}(x, t), \\
& p_{1}(s)=h_{3} p(t) / P, \\
& q_{1}\left(s^{\prime}\right)=h_{3} q(t) / P,
\end{aligned}
$$

Thus, the equilibrium conditions Equations (19) and (20) can be defined as:

$$
\begin{gathered}
\frac{a}{h_{3}} \int_{-1}^{1} p_{1}(s) d s=1, \\
\frac{b}{h_{3}} \int_{-1}^{1} q_{1}\left(s^{\prime}\right) d s^{\prime}=1 .
\end{gathered}
$$

Meanwhile, the singular integral Equations (17) and (18) can be simplified as:

$$
\begin{array}{r}
\int_{-1}^{1}\left(\frac{1}{s-r}+a \bar{K}_{1}(r, s)\right) p_{1}(s) d s+b e^{\beta_{2} h_{2}} \int_{-1}^{1} \bar{K}_{2}\left(r, s^{\prime}\right) q_{1}\left(s^{\prime}\right) d s^{\prime}=0,(|r| \leq 1), \\
a \int_{-1}^{1} \bar{K}_{3}\left(r^{\prime}, s\right) p_{1}(s) d s+e^{\beta_{2} h_{2}} \int_{-1}^{1}\left(\frac{1}{s^{\prime}-r^{\prime}}+b \bar{K}_{4}\left(r^{\prime}, s^{\prime}\right)\right) q_{1}\left(s^{\prime}\right) d s^{\prime}=0,\left(\left|r^{\prime}\right| \leq 1\right) .
\end{array}
$$

Following the method of Erdogan and Gupta [46], Equation (33) is of the type with singularity index " 1 ", since the singular behavior of the unknown function $p_{1}(s)$ appears while $s= \pm 1$, and Equation (34) is of the type with singularity index " -1 ", since the unknown function $q_{1}\left(s^{\prime}\right)$ is continuous and bounded throughout the closed interval $\left|s^{\prime}\right| \leq 1$. Thus, the flat rectangular indenter can be distinguished from the smooth indenters with differentiable shapes, including circular indenters, for the singularities existed in the tip of the flat rectangular indenter. Therefore, traditional methods for solving the singular integral equations for the receding contact problem with a circular indenter or a uniformly distributed load are inadequate here. This work suggests an innovative discretizing method for addressing the singularities as follows.

First, the integral portion that contains the top layer stationary contact pressure can be discretized using the Gauss-Chebyshev integral formulas of the first kind, and the integral portion that contains the bottom layer receding contact press can be discretized using the Gauss-Chebyshev integral formulas of the second kind. At the same time, Equations (31) and (33) are solved with Chebyshev polynomials of the second kind, and Equations (32) and (34) are solved with Chebyshev polynomials of the first kind. Now we can find:

$$
\sum_{k=1}^{N} \frac{1}{N}\left(\frac{1}{s^{\prime}{ }_{k}-r^{\prime}{ }_{j}}+a \bar{K}_{1}\left(r^{\prime}{ }_{j}, s^{\prime}{ }_{k}\right)\right) p_{2}\left(s^{\prime}{ }_{k}\right)+b e^{\beta_{2} h_{2}} \sum_{k=1}^{N} \frac{1-s_{k}^{2}}{N+1} \bar{K}_{2}\left(r^{\prime}{ }_{j}, s_{k}\right) q_{2}\left(s_{k}\right)=0,
$$




$$
\begin{gathered}
a \sum_{k=1}^{N} \frac{1}{N} \bar{K}_{3}\left(r_{j}, s^{\prime}{ }_{k}\right) p_{2}\left(s^{\prime}{ }_{k}\right)+e^{\beta_{2} h_{2}} \sum_{k=1}^{N} \frac{1-s_{k}^{2}}{N+1}\left(\frac{1}{s_{k}-r_{j}}+b \bar{K}_{4}\left(r_{j}, s_{k}\right)\right) q_{2}\left(s_{k}\right)=0, \\
\frac{\pi a}{h_{3}} \sum_{k=1}^{N} \frac{1}{N} p_{2}\left(s^{\prime}{ }_{k}\right)=1, \\
\frac{\pi b}{h_{3}} \sum_{k=1}^{N} \frac{1-s_{k}^{2}}{N+1} q_{2}\left(s_{k}\right)=1,
\end{gathered}
$$

in which:

$$
\begin{gathered}
p_{2}(s)=p_{1}(s)\left(1-s^{2}\right)^{1 / 2}, \\
q_{2}(s)=q_{1}(s)\left(1-s^{2}\right)^{-1 / 2}, \\
s_{k}=\cos \frac{\pi k}{N+1},(k=1,2, \cdots, N) ; \\
s^{\prime}{ }_{k}=\cos \frac{\pi(2 k-1)}{2 N},(k=1,2, \cdots, N) ; \\
r_{j}=\cos \frac{\pi(2 j-1)}{2(N+1)},(j=1,2, \cdots, N+1) ; \\
r_{j}^{\prime}=\cos \frac{\pi j}{N},(j=1,2, \cdots, N-1) .
\end{gathered}
$$

Obviously, there are $N+1$ equations derived from Equation (36), which correspond to $N+1$ different unknown variables $r_{j}$. Specifically, $N$ is an even number. The $(N / 2+1) t h$ equation is established automatically [47], and can be safely deleted. Thus, Equations (35)-(38) only contains $2 N+1$ equations, as well as $2 N+1$ unknowns, i.e., the contact pressure $p_{2}\left(s^{\prime}{ }_{k}\right), q_{2}\left(s_{k}\right),(k=1,2, \cdots, N)$ and the half-length of receding contact $b$. It is worth noting that, among all the unknowns in Equations (35)-(38), only $b$ is non-linear. We can initialize $b$ with a random value and substitute it into Equations (35)-(38); the remaining unknowns $p_{2}\left(s^{\prime}{ }_{k}\right)$ and $q_{2}\left(s_{k}\right),(k=1,2, \cdots, N)$ can then be solved iteratively. The numerical results of the iteration method can be verified by Equation (38), if the absolute error $\delta$ satisfies:

$$
|\delta|=\left|1-\frac{\pi b}{h_{3}} \sum_{k=1}^{N} \frac{1-s_{k}^{2}}{N+1} q_{2}\left(s_{k}\right)\right|<10^{-7} .
$$

\section{Numerical Results and Discussion}

In all numerical experiments, Poisson's ratios are fixed as $v=0.25,(\kappa=2)$. Several well-studied models can be expressed by the presented study if the key parameters are modified as specific values. For instance, this study can be reduced to a receding contact problem between a homogeneous layer and a half-plane under a concentrated force, if the parameters satisfy: $\beta_{3} h_{3}=0.001, h_{2}=0$ and $a / h_{3}=0.01$. Our simulation results show that the corresponding normalized receding contact half-length is 1.3243 , which is almost identical to the results reported by the early literature $[48,49]$. The accuracy of the numerical results can thus be verified.

This work first investigates the receding contact distributions for different indenter sizes. Both Figures 2 and 3 show positive relationships between the indenter size and the stationary/receding contact half-length, which means a larger indenter size invariably leads to a larger stationary/receding contact half-length. Correspondingly, the minimum stationary contact pressure and the maximum receding contact pressure are both negatively correlated with the indenter size. Comparing the position in which the extreme value appears, it can be determined that the minimum stationary contact pressure always appears at the center of the contact area, while the maximum receding contact pressure does not deterministically appear at the similar position. For several cases, a pressure concave 
may appear at the center of the receding contact area, and the larger the receding contact half-length, the more obvious the pressure concave.

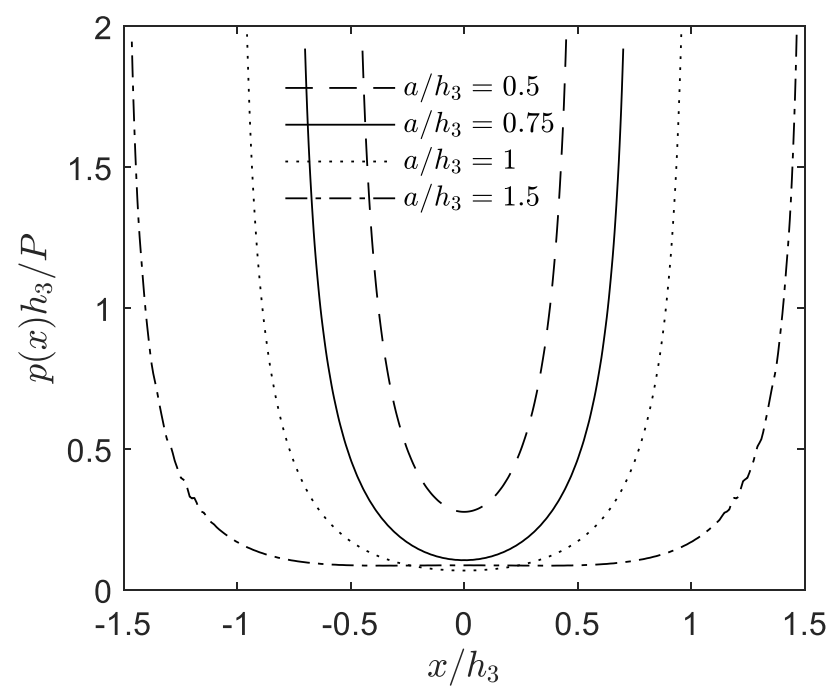

Figure 2. The effect of a non-dimensionalized indenter size $a / h_{3}$ on the distribution of nondimensionalized stationary contact pressure $\left(\mu_{03}=\mu_{1}, h_{2}=h_{3}, \beta_{2} h_{2}=\beta_{3} h_{3}=1\right)$.

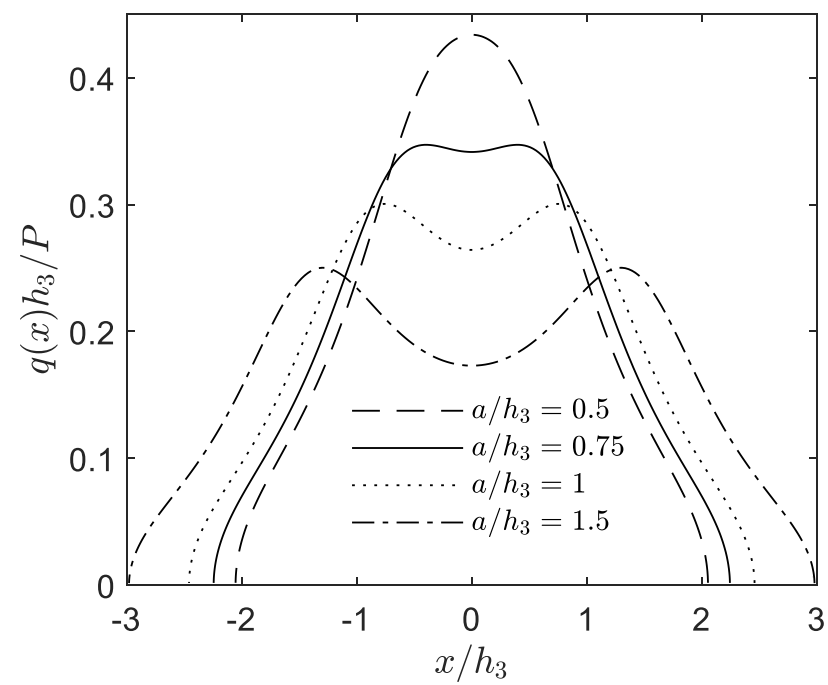

Figure 3. The effect of a non-dimensionalized indenter size $a / h_{3}$ on the distribution of nondimensionalized receding contact pressure $\left(\mu_{03}=\mu_{1}, h_{2}=h_{3}, \beta_{2} h_{2}=\beta_{3} h_{3}=1\right)$.

Figures 4 and 5 reflect the regulation effects on the non-dimensionalized receding contact half-length caused by the inhomogeneity parameters $\beta_{3} h_{3}$ and $\beta_{2} h_{2}$. The numerical examples in these two figures show that the width of the flat indenter is positively correlated with the non-dimensionalized receding contact half-length. In detail, a non-monotonic function appears in Figure 4 which describes the correlation between the inhomogeneity parameter of the top layer and the non-dimensionalized receding contact half-length. Along with the increasing of the inhomogeneity parameter, the non-dimensionalized receding contact half-length first decreases, and then increases, while the non-dimensionalized receding contact half-length monotonically decreases for the inhomogeneity parameter of the interlayer in Figure 5. Specially, Figure 5 shows the receding contact half-lengths between the homogeneous elastic layer and the FG coated homogeneous half-plane due to the flat indenters. Comparing Figure 5 with the 7th figure of reference [45], we can also investigate the interaction between the shape of the indenter and the inhomogeneity parameter of the FG coating. Regardless of shape (circular or flat) of the indenter, the 
receding contact half-length decreases with the increases in the inhomogeneity parameters of the FG coating, as well as the decrease in the indenter size. However, under the flat indenter, the regulations of the inhomogeneity parameters of the FG coating on the receding contact half-length are less efficient than those under the circular indenter.

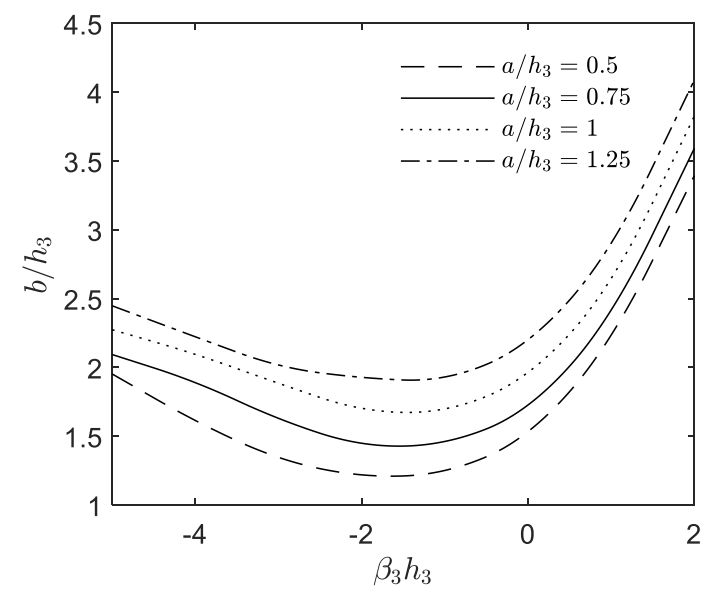

Figure 4. The effects of inhomogeneity parameter $\beta_{3} h_{3}$ on the non-dimensionalized receding contact half-length $\left(\mu_{03}=\mu_{1}, h_{2}=h_{3}, \beta_{2} h_{2}=0.001\right)$.

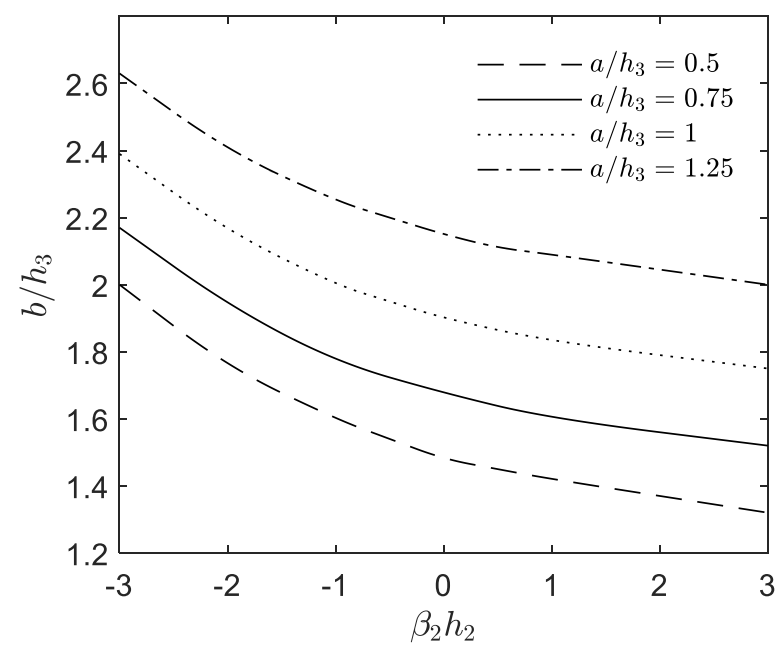

Figure 5. The effects of the inhomogeneity parameter $\beta_{2} h_{2}$ on the non-dimensionalized receding contact half-length $\left(\mu_{03}=\mu_{1}, h_{2}=h_{3}, \beta_{2} h_{2}=0.001\right)$.

For the monotony correlation exhibited in Figure 5, the way in which the inhomogeneity parameter of the interlayer $\beta_{2} h_{2}$ influences the contact pressure should be investigated further. Figure 6 illustrates the distribution of the stationary contact pressure located between the indenter and the FG layer. The stationary contact half-length depends only on the indenter size; any other parameters cannot influence it. Figure 6a shows the minimum contact pressure achieved at the center of the stationary contact area. At the edge of the area, the contact pressure increases sharply for the singularity, which occurs at the border of the indenter. It is difficult to estimate the influence of the inhomogeneity parameter on the stationary contact pressure since the contact pressure at the edge area is too large. The details are shown in Figure $6 \mathrm{~b}, \mathrm{c}$. A noteworthy phenomenon is also indicated by Figure $6 \mathrm{~b}, \mathrm{c}$ : a positive relationship emerges between the inhomogeneity parameter of the interlayer and the contact pressure of the stationary contact center area, while the relationship near the edge of the stationary contact is the reverse. 

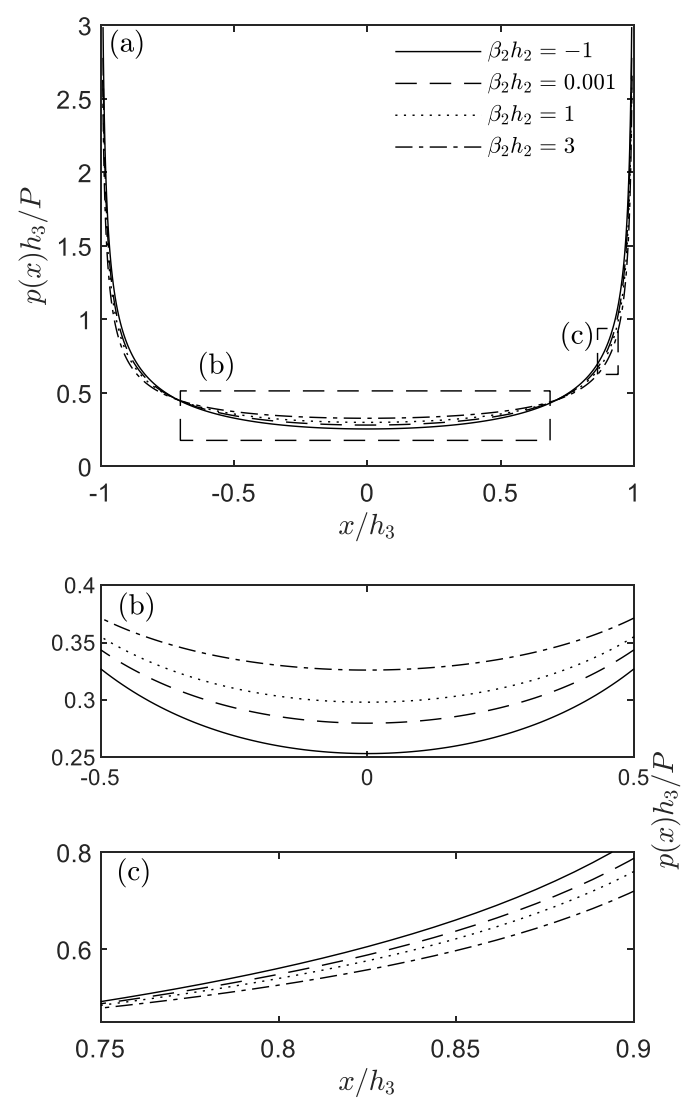

Figure 6. (a) The effects of the inhomogeneity parameter $\beta_{2} h_{2}$ on the distribution of the nondimensionalized stationary contact pressure, with details near (b) the stationary contact center and (c) the boundaries magnified $\left(\mu_{03}=\mu_{1}, h_{2}=h_{3}, \beta_{3} h_{3}=0.001, a / h_{3}=0.5\right)$.

Figure 7 shows the distribution of the non-dimensionalized receding contact pressure for different inhomogeneity parameters of the interlayer. There is an obvious inverse correlation between the maximum value of the receding contact pressure and the receding contact half-length. The influence of the inhomogeneity parameter $\beta_{2} h_{2}$ on the receding contact pressure is similar to that on the stationary contact pressure. The maximum value of the receding contact pressure increases with the increase in the inhomogeneity parameter $\beta_{2} h_{2}$.

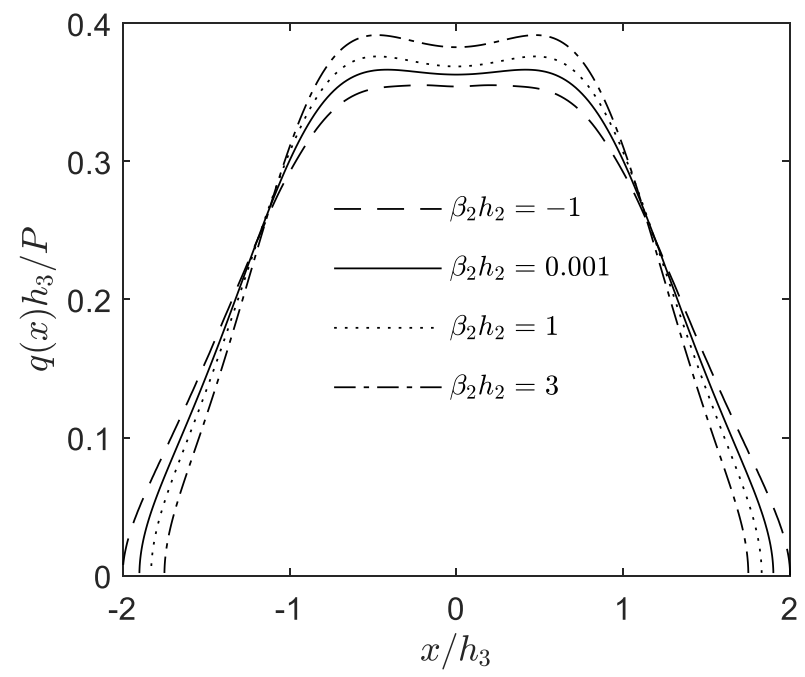

Figure 7. The effects of the inhomogeneity parameter $\beta_{2} h_{2}$ on the distribution of the nondimensionalized receding contact pressure $\left(\mu_{03}=\mu_{1}, h_{2}=h_{3}, \beta_{3} h_{3}=0.001, a / h_{3}=0.5\right)$. 
Figure 8 illustrates how the shear moduli of both FG layers' bottom surfaces regulate the normalized receding contact half-length. For any inhomogeneity parameter value, a larger shear modulus value of the top layer's bottom surface always corresponds to a smaller receding contact size and to a larger maximum value of receding contact pressure as well. By combining Figures 4 and 5, we observe an interesting effect: while $\beta_{2} h_{2}=\beta_{3} h_{3}>-1$, the influence on the receding contact half-length caused by the inhomogeneity of the top FG layer is larger than that caused by the inhomogeneity of the interlayer.



Figure 8. The dependencies of the non-dimensionalized receding contact half-length on the shear moduli ratio $\mu_{03} / \mu_{1}$ for different inhomogeneity parameters $\beta_{2} h_{2}$ and $\beta_{3} h_{3}\left(h_{2}=h_{3}, a / h_{3}=0.5\right)$.

The thickness of the interlayer can also regulate the receding contact half-length. Figure 9 focuses on the influence of the interlayer's thickness on the receding contact halflength, while the inhomogeneity parameters of the top layer and the interlayer are equal. For a special case $\beta_{2} h_{2}=\beta_{3} h_{3}=0.001$, the proposed model is reduced to a receding contact problem between a homogeneous layer and a homogeneous half-plane. The thickness of the homogeneous interlayer does not significantly influence the contact area. This indicates that a harder and thicker FG interlayer results in a large amount of receding contact, and therefore, a reduced peak contact pressure in Figure 9. The opposite is true for a soft FG interlayer. The thicker the soft FG interlayer, the smaller the receding contact half-length. This also leads to an increase in the maximum contact pressure.

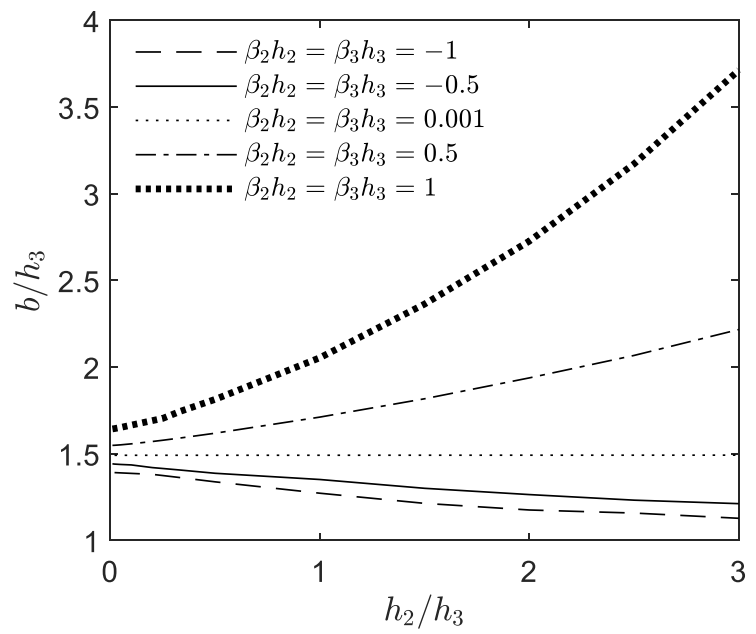

Figure 9. The dependence of non-dimensionalized receding contact half-length on the thickness ratio $h_{2} / h_{3}$ for different inhomogeneity parameters $\beta_{2} h_{2}$ and $\beta_{3} h_{3}\left(\mu_{03}=\mu_{1}, a / h_{3}=0.5\right)$. 
Figure 10 focuses on the influence of the interlayer's thickness on the receding contact half-length, while the inhomogeneity parameters of the top layer and the interlayer are not equal. However, the combined effect of the thickness of the FG interlayer and the inhomogeneity parameters is difficult to determine from Figure 10. The manifested features in Figure 10 are similar to those in Figure 9, but the combined effect of the thickness of the FG interlayer and the inhomogeneity parameters is still ambiguous in this figure.

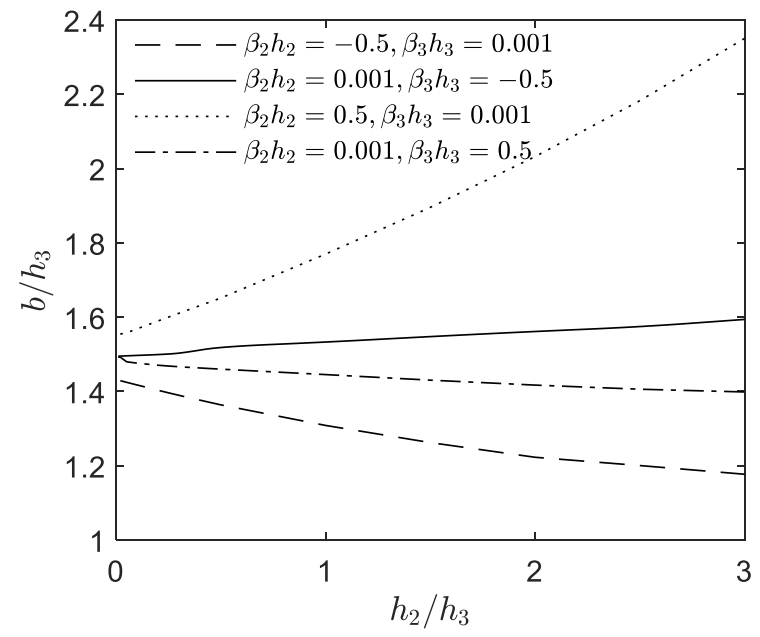

Figure 10. The dependence of the non-dimensionalized receding contact half-length on the thickness ratio $h_{2} / h_{3}$ for different inhomogeneity parameters $\beta_{2} h_{2}$ and $\beta_{3} h_{3}\left(\mu_{03}=\mu_{1}, a / h_{3}=0.5\right)$.

\section{Conclusions}

In this study, the receding contact problem of a multi-layered elastic structure involving FGM under a flat indenter is considered. The model is transformed into dual singular integral equations that are then solved numerically. From comprehensive parametric research studying the inhomogeneity parameters of FGM, the indenter size, the interlayer's thickness, and the material properties, we can finally come to a number of noteworthy conclusions:

The stationary contact size is only related to the flat indenter size. A wider flat indenter produces a larger stationary contact size. As a result, the pressure in the stationary contact center area is reduced.

The center of the receding contact area sustains the maximum pressure while the receding contact half-length is small. However, when the half-length is large enough, a special pressure concave may appear at the center of the contact area. The maximum receding contact pressure is inversely related to the receding contact half-length; the longer the half-length, the deeper the pressure concave.

The receding contact half-length is inversely correlated with the inhomogeneity parameter of the interlayer and the shear modulus ratio is $\mu_{03} / \mu_{1}$; it is positively correlated with the indenter size.

The interlayer thickness can regulate the receding contact area, but the regulation effect is also influenced by the inhomogeneity parameters $\beta_{2} h_{2}$ and $\beta_{3} h_{3}$.

Author Contributions: Conceptualization, J.Y. and C.W.; methodology, J.Y.; software, C.W.; validation, J.Y.; writing - original draft preparation, J.Y.; writing-review and editing, C.W. All authors have read and agreed to the published version of the manuscript.

Funding: This research was funded by the National Natural Science Foundation of China, grant number 61602331, and the Opening Project of Intelligent Policing Key Laboratory of Sichuan Province, grant number ZNJW2022ZZMS002\&ZNJW2022ZZMS004.

Institutional Review Board Statement: Not applicable.

Informed Consent Statement: Not applicable. 
Data Availability Statement: Not applicable.

Conflicts of Interest: The authors declare no conflict of interest. The funders had no role in the design of the study; in the collection, analyses, or interpretation of data; in the writing of the manuscript, or in the decision to publish the results.

\section{Appendix A}

The expressions of $K_{j}(x, t)(j=1,2,3,4)$ appearing in Equations (17) and (18) are given by:

$$
\begin{array}{r}
K_{1}(x, t)=\int_{0}^{\infty}\left[\frac{\lambda}{d_{1}} \sum_{k=1}^{4}(-1)^{k} \frac{B_{1 k}}{B} n_{k 2}(\lambda) e^{m_{k 2} h_{2}}-1\right] \sin [\lambda(t-x)] d \lambda, \\
K_{2}(x, t)=\int_{0}^{\infty} \frac{\lambda}{d_{1}} \sum_{k=1}^{4}(-1)^{k} \frac{B_{3 k}}{B} n_{k 2}(\lambda) e^{m_{k 2} h_{2}} \sin [\lambda(t-x)] d \lambda, \\
K_{3}(x, t)=\int_{0}^{\infty} \frac{\lambda}{d_{2}} \sum_{k=1}^{4}(-1)^{k} \frac{B_{1 k}}{B} n_{k 2}(\lambda) \sin [\lambda(t-x)] d \lambda, \\
K_{4}(x, t)=\int_{0}^{\infty}\left[\frac{\lambda}{d_{2}} \sum_{k=1}^{4}(-1)^{k}\left(\frac{B_{3 k}}{B} n_{k 2}(\lambda)-\frac{\mu_{03} e^{\beta_{3} h_{2}} A_{3 k}}{\mu_{1} e^{\beta_{2} h_{2}} A} n_{k 1}(\lambda)\right)-1\right] \sin [\lambda(t-x)] d \lambda,
\end{array}
$$

where:

$$
\begin{gathered}
d_{1}=-\frac{\kappa+1}{4}, \\
d_{2}=\frac{\kappa+1}{4}\left(1+\frac{\mu_{03} e^{\beta_{3} h_{2}}}{\mu_{1} e^{\beta_{2} h_{2}}}\right)
\end{gathered}
$$

$A_{3 k}(k=1,2,3,4)$ are the sub-determinants of determinants $A$ which dropped the third row and the $k$-th column. Similarly, $B_{j k}(j=1,3 ; k=1,2,3,4)$ represents the subdeterminants of determinants $B$ without the $j$-th row and the k-th column. Expression for the matrix $A$ and $B$ are given by:

$$
\begin{gathered}
A=\left[\begin{array}{ccccc}
\Delta_{11} e^{m_{11} h} & \Delta_{21} e^{m_{21} h} & \Delta_{31} e^{m_{31} h} & \Delta_{41} e^{m_{41} h} \\
\Lambda_{11} e^{m_{11} h} & \Lambda_{21} e^{m_{21} h} & \Lambda_{31} e^{m_{31} h} & \Lambda_{41} e^{m_{41} h} \\
\Delta_{11} e^{m_{11} h} & \Delta_{21} e^{m_{21} h} & \Delta_{31} e^{m_{31} h} & \Delta_{41} e^{m_{41} h} \\
\Lambda_{11} e^{m_{11} h} & \Lambda_{21} e^{m_{21} h} & \Lambda_{31} e^{m_{31} h} & \Lambda_{41} e^{m_{41} h}
\end{array}\right], \\
B=\left[\begin{array}{cccccc}
\Delta_{12} e^{m_{12} h_{2}} & \Delta_{22} e^{m_{12} h_{2}} & \Delta_{32} e^{m_{32} h_{2}} & \Delta_{42} e^{m_{42} h_{2}} & 0 & 0 \\
\Lambda_{12} e^{m_{12} h_{2}} & \Lambda_{22} e^{m_{22} h_{2}} & \Lambda_{32} e^{m_{32} h_{2}} & \Lambda_{42} e^{m_{42} h_{2}} & 0 & 0 \\
\Delta_{12} & \Delta_{22} & \Delta_{32} & \Delta_{42} & -2 i \lambda & (1+\kappa) i \\
\Lambda_{12} & \Lambda_{22} & \Lambda_{32} & \Lambda_{42} & -2 \lambda & \kappa-1 \\
1 & 1 & 1 & 1 & -1 & 0 \\
n_{12} & n_{22} & n_{32} & n_{42} & -i & \kappa i / \lambda
\end{array}\right],
\end{gathered}
$$

where:

$$
\begin{gathered}
h=h_{1}+h_{2}, \\
\Delta_{k 1}=-i(3-\kappa) \lambda+(1+\kappa) m_{k 1} n_{k 1} /(\kappa-1),(k=1,2,3,4), \\
\Lambda_{k 1}=m_{k 1}-i \lambda n_{k 1},(k=1,2,3,4), \\
\Delta_{k 2}=-i(3-\kappa) \lambda+(1+\kappa) m_{k 2} n_{k 2} /(\kappa-1),(k=1,2,3,4), \\
\Lambda_{k 2}=m_{k 2}-i \lambda n_{k 2},(k=1,2,3,4), \\
m_{1 j}=\overline{m_{3 j}},
\end{gathered}
$$




$$
\begin{gathered}
m_{2 j}=\overline{m_{4 j}}, \\
m_{3 j}=\frac{1}{2}\left(-\beta_{j}+\sqrt{\beta_{j}^{2}+4 \lambda^{2}+(-1)^{j} 4 i \beta_{j} \lambda \sqrt{(3-\kappa) /(1+\kappa)}}\right), \\
m_{4 j}=\frac{1}{2}\left(-\beta_{j}-\sqrt{\beta_{j}^{2}+4 \lambda^{2}+(-1)^{j} 4 i \beta_{j} \lambda \sqrt{(3-\kappa) /(1+\kappa)}}\right), \\
n_{k j}=\frac{(\kappa-1) m_{k j}^{2}+\beta_{j}(\kappa-1) m_{k j}-\lambda^{2}(\kappa+1)}{i \lambda\left(2 m_{k j}+\beta_{j}(\kappa-1)\right)},(k=1,2,3,4 ; j=1,2),
\end{gathered}
$$

additionally, it has $i^{2}=-1$.

\section{References}

1. Comez, I.; Birinci, A.; Erdol, R. Double receding contact problem for a rigid stamp and two elastic layers. Eur. J. Mech. A Solids 2004, 23, 301-309. [CrossRef]

2. Cakiroglu, E.; Coemez, I.; Erdoel, R. Application of artificial neural networks to a double receding contact problem with a rigid stamp. Struct. Eng. Mech. 2005, 21, 205-220. [CrossRef]

3. Adibelli, H.; Comez, I.E. Receding contact problem for a coated layer and a half-plane loaded by a rigid cylindrical stamp. Arch. Mech. 2013, 65, 219-236.

4. Yaylaci, M.; Birinci, A. The receding contact problem of two elastic layers supported by two elastic quarter planes. Struct. Eng. Mech. 2013, 48, 241-255. [CrossRef]

5. Yaylac, M.; Öner, E.; Birinci, A. Comparison between Analytical and ANSYS Calculations for a Receding Contact Problem. J. Eng. Mech. 2014, 140, 04014070. [CrossRef]

6. Yaylaci, E.U.; Yaylaci, M.; Olmez, H.; Birinci, A. Artificial neural network calculations for a receding contact problem. Comput. Concr. 2020, 25, 551-563.

7. Ke, L.L.; Wang, Y.S. Two-dimensional contact mechanics of functionally graded materials with arbitrary spatial variations of material properties. Int. J. Solids Struct. 2006, 43, 5779-5798. [CrossRef]

8. Liu, T.J.; Zhang, C.; Wang, Y.S.; Xing, M.-G. The axisymmetric stress analysis of double contact problem for functionally graded ma-terials layer with arbitrary graded materials properties. Int. J. Solids Struct. 2016, 96, 229-239. [CrossRef]

9. Comez, I.; Kahya, V.; Erdol, R. Plane receding contact problem for a functionally graded layer supported by two quarter-planes. Arch. Mech. 2018, 70, 485-504.

10. Adıyaman, G.; Birinci, A.; Öner, E.; Adıyaman, G.; Birinci, A.; Öner, E.; Yaylacı, M. A receding contact problem between a functionally graded layer and two homogeneous quarter planes. Acta Mech. 2016, 227, 1753-1766. [CrossRef]

11. Chen, S.; Cong, Y.; Soh, A. Adhesive behavior of two-dimensional power-law graded materials. Int. J. Solids Struct. 2009, 46, 3398-3404. [CrossRef]

12. Fan, J.; Xu, G.; Gao, H. Adhesive contact on power-law graded elastic solids: The JKR-DMT transition using a double-Hertz model. J. Mech. Phys. Solids 2013, 61, 2473-2492.

13. Erdogan, F. Fracture mechanics of functionally graded materials. Compos. Eng. 1995, 5, 753-770. [CrossRef]

14. Boussoula, A.; Boucham, B.; Bourada, M.; Bourada, F.; Tounsi, A.; Bousahla, A.A.; Tounsi, A. A simple nth-order shear deformation theory for thermomechanical bending analysis of different configurations of FG sandwich plates. Smart Struct. Syst. 2020, 25, 197-218.

15. Kaddari, M.; Kaci, A.; Bousahla, A.A.; Tounsi, A.; Bourada, F.; Tounsi, A.; Adda Bedia, E.A.; Al-Ostra, L.A. A study on the structural behaviour of functionally graded porous plates on elas-tic foundation using a new quasi-3D model: Bending and free vibration analysis. Comput. Concr. 2020, 25, 37-57.

16. Kieback, B.; Neubrand, A.; Riedel, H. Processing techniques for functionally graded materials. Mater. Sci. Eng. A 2003, 362, 81-106. [CrossRef]

17. Luo, Y.W.; Ma, T.; Shao, W.W.; Zhang, G.B.; Zhang, B. Effects of heat treatment on microstructures and mechanical properties of gh4169/k418 functionally graded material fabricated by laser melting deposition. Mater. Sci. Eng. 2021, 821, 141601. [CrossRef]

18. Namigata, S.; Tsukamoto, H. Fabrication of carbon nanotube/aluminum matrix functionally graded materials using centrif-ugal slurry methods. Key Eng. Mater. 2021, 878, 31-40. [CrossRef]

19. Krumova, M.; Klingshirn, C.; Haupert, F.; Friedrich, K. Microhardness studies on functionally graded polymer composites. Compos. Sci. Technol. 2001, 61, 557-563. [CrossRef]

20. Chen, D.Q.; Sun, D.L.; Li, X.F. Surface effects on resonance frequencies of axially functionally graded Timoshenko nanocanti-levers with attached nanoparticle. Compos. Struct. 2017, 173, 116-126. [CrossRef]

21. Rk, A.; Sk, A.; Dkp, A.; Gupta, S. Green's function and surface waves in a viscoelastic orthotropic FGM enforced by an impulsive point source. Appl. Math. Comput. 2020, 382, 125325. 
22. Hong, Y.; Neu, R.W.; Berto, F.; Endo, M. Fatigue \& fracture of engineering materials \& structures. Fatig. Fract. Eng. Mat. Struct. 2018, 41, 247.

23. Ammendolea, D.; Greco, F.; Lonetti, P.; Luciano, R.; Pacuzza, R. Crack propagation modeling in functionally graded materials using moving mesh technique and interaction integral approach. Compos. Struct. 2021, 269, 114005. [CrossRef]

24. Singh, S.K.; Singh, I.V. Extended isogeometric analysis for fracture in functionally graded magneto-electro-elastic material. Eng. Fract. Mech. 2021, 247, 107640. [CrossRef]

25. Perkowski, D.M.; Kulchytsky-Zhyhailo, R.; Kolodziejczyk, W. On axisymmetric heat conduction problem for multilayer graded coated half-space. J. Teoret. Appl. Mech. 2018, 56, 147-156. [CrossRef]

26. Martínez-Pañeda, E. On the Finite Element Implementation of Functionally Graded Materials. Materials 2019, 12, 287. [CrossRef]

27. Ebrahimijahan, A.; Dehghan, M.; Abbaszadeh, M. Simulation of plane elastostatic equations of anisotropic functionally graded materials by integrated radial basis function based on finite difference approach. Eng. Anal. Bound. Elem. 2022, 134, 553-570. [CrossRef]

28. Choi, H.J.; Paulino, G.H. Thermoelastic contact mechanics for a flat punch sliding over a graded coating/substrate system with frictional heat generation. J. Mech. Phys. Solids 2008, 56, 1673-1692. [CrossRef]

29. Ke, L.-L.; Yang, J.; Kitipornchai, S.; Wang, Y.-S. Frictionless contact analysis of a functionally graded piezoelectric layered half-plane. Smart Mater. Struct. 2008, 17, 025003. [CrossRef]

30. Chen, P.; Chen, S. Partial slip contact between a rigid punch with an arbitrary tip-shape and an elastic graded solid with a finite thickness. Mech. Mater. 2013, 59, 24-35. [CrossRef]

31. Mez, I.; El-Borgi, S.; Kahya, V.; Erdol, R. Receding contact problem for two-layer functionally graded media indented by a rigid punch. Acta Mech. 2016, 227, 2493-2504.

32. Yilmaz, K.B.; Comez, I.; Yildirim, B.; Guler, M.A.; El-Borgi, S. Frictional Receding Contact Problem for a Graded Bilayer System Indented by a Rig-id Punch. Int. J. Mech. Sci. 2018, 141, 127-142. [CrossRef]

33. Yan, J.; Mi, C. On the receding contact between an inhomogeneously coated elastic layer and a homogeneous half-plane. Mech. Mater. 2017, 112, 18-27. [CrossRef]

34. Yan, J.; Mi, C. Double contact analysis of multilayered elastic structures involving functionally graded materials. Arch. Mech. 2017, 69, 199-221.

35. Liu, Z.; Yan, J.; Mi, C. On the receding contact between a two-layer inhomogeneous laminate and a half-plane. Struct. Eng. Mech. 2018, 66, 329-341.

36. Yan, J.; Mi, C. A receding contact analysis for an elastic layer reinforced with a functionally graded coating and pressed against a half-plane. J. Mech. Sci. Technol. 2019, 33, 4331-4344. [CrossRef]

37. Mez, I.; El-Borgi, S.; Yildirim, B. Frictional receding contact problem of a functionally graded layer resting on a homogeneous coated half-plane. Arch. Appl. Mech. 2020, 90, 2113-2131.

38. Yaylac, M.; Eyübolu, A.; Adyaman, G.; Yaylaci, E.U.; Oner, E.; Birinci, A. Assessment of different solution methods for receding contact problems in func-tionally graded layered mediums. Mech. Mater. 2021, 154, 103730. [CrossRef]

39. Oner, E.; Şengül, B.Ş.; Yaylacı, E.U.; Adyiaman, G.; Yaylaci, M.; Birinci, A. On the plane receding contact between two functionally graded layers using com-putational, finite element and artificial neural network methods. J. Appl. Math. Mech. 2022, 102, e202100287.

40. Karabulut, P.M.; Adiyaman, G.; Birinci, A. A receding contact problem of a layer resting on a half plane. Struct. Eng. Mech. 2017, 64, 505-513.

41. Ozsahin, T.S.; Taskiner, O. Contact Problem for an Elastic Layer on an Elastic Half Plane Loaded by Means of Three Rigid Flat Punches. Math. Probl. Eng. 2013, 4, 112-128. [CrossRef]

42. Cao, R.; Li, L.; Li, X.; Mi, C. On the frictional receding contact between a graded layer and an orthotropic substrate indented by a rigid flat-ended stamp. Mech. Mater. 2021, 158, 103847. [CrossRef]

43. Cao, R.; Mi, C. On the receding contact between a graded and a homogeneous layer due to a flat-ended indenter. Math. Mech. Solids 2021. [CrossRef]

44. Ke, L.L.; Wang, Y.-L. Two-dimensional contact mechanics and fretting analysis of functionally graded materials. Eur. J. Mech. 2007, 26, 171-188. [CrossRef]

45. Yan, J.; Mi, C. On the Receding Contact Between a Homogeneous Elastic Layer and a Half-plane Substrate Coated with Func-tionally Graded Materials. Int. J. Comput. Method. 2020, 17, 89-94. [CrossRef]

46. Erdogan, F.; Gupta, G.D. On the numerical solution of singular integral equations. Q. Appl. Math. 1972, 29, 525-534. [CrossRef]

47. Erdogan, F.; Gupta, G.D.; Cook, T.S. Numerical Solution of Singular Integral Equations; Springer: Berlin/Heidelberg, Germany, 1973

48. Keer, L.M.; Dundurs, J.; Tsai, K.C. Problems Involving a Receding Contact Between a Layer and a Half Space. J. Appl. Mech. 1972, 39, 1115-1120. [CrossRef]

49. El-Borgi, S.; Abdelmoula, R.; Keer, L. A receding contact plane problem between a functionally graded layer and a homogene-ous substrate. Int. J. Solids Struct. 2006, 43, 658-674. [CrossRef] 